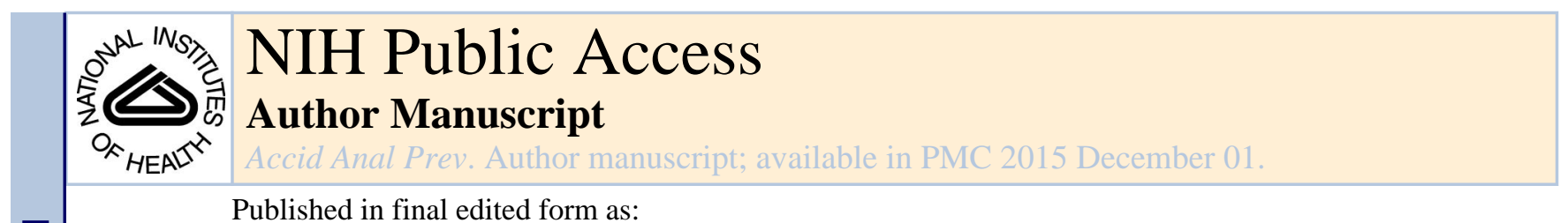

Published in final edited form as:

Accid Anal Prev. 2014 December ; 0: 373-379. doi:10.1016/j.aap.2014.09.026.

\title{
Ambulance Transport Rates after Motor Vehicle Collision for Older vs. Younger Adults: A Population-Based Study
}

\author{
Katherine M. Hunold, BSPH, Mark R. Sochor, MD, MS, Samuel A. McLean, MD, MPH, \\ Kaitlyn B. Mosteller, Antonio R. Fernandez, PhD, NREMT-P, and Timothy F. Platts-Mills, \\ MD, MSc \\ University of North Carolina at Chapel Hill, Department of Emergency Medicine, 170 Mannning \\ Drive, CB 7594, Chapel Hill, NC, 27599-7594, United States
}

\begin{abstract}
Older adults are at greater risk than younger adults for life-threatening injury after motor vehicle collision (MVC). Among those with life-threatening injury, older adults are also at greater risk of not being transported by emergency medical services (EMS) to an emergency department. Despite the greater risk of serious injury and non-transportation among older adults, little is known about the relationship between patient age and EMS transportation rates for individuals experiencing MVC. We describe transport rates across the age-span for adults seen by EMS after experiencing MVC using data reported to the North Carolina Department of Motor Vehicles between 2008 and 2011. Of all adults aged 18 years and older experiencing MVC and seen by EMS ( $\mathrm{N}=484,310)$, $36.3 \%(\mathrm{~N}=175,768)$ were transported to an emergency department. Rates of transport for individuals seen by EMS after MVC increased only a small amount with increasing patient age. After adjusting for potential confounders of the relationship between patient age and the decision to transport (patient gender, patient race, air bag deployment, patient trapped or ejected, and injury severity), transport rates were: age 18-64 = 36.0\% (95\% Confidence Interval [CI], 35.9\%-36.2\%); age $65-74=36.6 \%$ (95\% CI, 36.0\%-37.1\%); age 75-84 = 37.3\% (95\% CI, 36.5\%-38.1\%), and age 85-94 $=38.2 \%$ (95\% CI, 36.7\%-39.8\%). In North Carolina between 2008 and 2011, the transportation rate was only slightly higher for older adults than for younger adults, and most older adults experiencing MVC and seen by EMS were not transported to the emergency department. These findings have implications for efforts to improve the sensitivity of criteria used by EMS to determine the need for transport for older adults experiencing MVC.
\end{abstract}

\section{Keywords}

geriatrics; motor vehicle collision; emergency medical services; triage

(C) 2014 Elsevier Ltd. All rights reserved.

Corresponding Author: Dr. Platts-Mills F. Timothy, MD, MSc, University of North Carolina at Chapel Hill.

The content is solely the responsibility of the authors and does not necessarily represent the official views of the National Institutes of Health or the North Carolina Department of Transportation.

Publisher's Disclaimer: This is a PDF file of an unedited manuscript that has been accepted for publication. As a service to our customers we are providing this early version of the manuscript. The manuscript will undergo copyediting, typesetting, and review of the resulting proof before it is published in its final citable form. Please note that during the production process errors may be discovered which could affect the content, and all legal disclaimers that apply to the journal pertain. 


\section{INTRODUCTION}

More than 10 million individuals are involved in motor vehicle collisions (MVCs) each year in the United States ${ }^{1}$. Approximately 3.4-3.9 million of these individuals receive treatment in an emergency department (ED) and approximately 30,000 (0.3\%) die as a result of the $\mathrm{MVC}^{2}$. Adults aged 65 years and older compose an estimated 250,000 of these MVC-related ED visits ${ }^{3}$, and are at greater risk than younger adults for debilitating injury or death ${ }^{4}$. Due to the anticipated increase in the number of older drivers, the number of older adults involved in MVCs is expected to double by $2030^{5}$.

The prehospital assessment of individuals experiencing MVC is an important component of the care for these patients ${ }^{6}$. In the US, there are no national guidelines defining which individuals require transportation to a hospital after MVC. Instead, in most EMS systems this decision is based on the judgment of the EMS provider based on their clinical assessment, local or state protocols, and at times also influenced by patient preference. Inappropriate destinations and non-transportation of older adults with significant injuries after trauma have been reported in multiple trauma systems and linked to worse outcomes $^{7-12}$. Studies of non-transportation of individuals experiencing MVC indicate that approximately $10 \%$ of older adults seen by EMS but not transported will later seek ED care and $30 \%$ of these patients are subsequently admitted to the hospital ${ }^{8,13}$. Death among those experiencing MVC and evaluated but not transported by EMS is rare but more common among older than younger adults ${ }^{8}$.

Understanding the epidemiology of EMS transports after MVC is an essential step to addressing the problem of non-transportation of older patients with serious or lifethreatening injuries ${ }^{14}$. First, understanding the relationship between patient age and EMS transport rates will provide insight into current patterns of EMS provider decision making. Second, one potential solution to the problem of non-transportation of older adults with serious injuries after MVC is revised guidelines in which the threshold for transportation is explicitly lower for older adults ${ }^{14}$. However, the impact of altering guidelines for the transportation of older adults experiencing MVC depends on existing behavior. If the current transport rate for these older adults is low, such changes could add substantially to both the financial costs and human resource required to provide emergency care for this population. In contrast, if most older adults are already transported, such a requirement would add little to the cost of prehospital care.

The purpose of this study is to describe EMS transport rates for age groups of adults experiencing MVC with particularly attention to older adults. We hypothesized that most older adults seen by EMS after MVC would be transported and that this transport rate would be $m$ much higher than for younger adults.

\section{METHODS}

\section{Study Design and Population}

We analyzed cross-sectional data from the North Carolina Department of Motor Vehicles (NC DMV) CRASH database, an administrative dataset recording information about all 
MVCs across the state of North Carolina. Data were analyzed for calendar years 2008-2011, the four most recent years for which data were available. This dataset describes each MVC as recorded at the collision scene by a NC Law Enforcement Agent using the NC DMV's Crash Report Form (DMV-349). Most of the forms are completed electronically; the remainder are completed on paper and entered by NC DMV staff. The NC DMV validates the data for completeness and logical entries. MVCs are required to be reported if there is $\$ 1,000$ or more in damage or injury to any person involved; MVCs below this threshold that are reported to the DMV are also included.

Individuals involved in an MVC were included in the analysis if they were between the ages of 18 and 94 years. Patients aged 95 years and older were excluded from analysis because the estimates of transport rates for these individuals were unreliable due to small sample size. Individuals who died as a result of the MVC were included in the analysis. Analysis focused on those individuals who were seen by EMS, defined as all individuals involved in an MVC for which EMS was known to be present. Prehospital care in North Carolina is provided by an estimated 22,900 active EMS personnel working in 433 EMS agencies. The study was exempted from formal review by the institutional review board at The University of North Carolina at Chapel Hill.

\section{Age Categories}

For the primary analysis, age was analyzed in four categories: $18-64,65-74,75-84$, and $85-94$ years. In a secondary analysis, age was divided into 39 groups $(18-19,20-21,22-23$, etc.). Categorical analysis of age was chosen to allow the identification of non-linear trends.

\section{Collision and Victim Characteristics}

MVC characteristics described on the collision level included number of cars involved, number of non-motorists involved, crash locality, road surface condition, whether or not weather contributed to the MVC, ambient light, whether or not alcohol or drugs were a contributing factor, damage severity, damage cost estimate, and whether or not EMS responded to the MVC. These variables are reported with means and standard deviations for continuous variables and numbers and percentages for categorical variables. In addition, speed at impact was utilized in sensitivity analysis. Information described on the individual level included victim age, ethnicity, gender, air bag deployment, whether the person was trapped or ejected, injury severity including death, whether EMS was on the scene of the collision, and if the person was transported by EMS.

\section{Outcome Measure}

The primary outcome was the whether an individual was transported by EMS. This outcome was reported by age groups both without adjustment and adjusted for covariates which might confound the relationship between age and EMS transport.

\section{Data Analysis}

The primary analysis examined EMS transports rates among patients seen by EMS after MVC by the four age groups (18-64, 65-74, 75-84, and 85-94). Logistic regression was used to obtain adjusted rates of transportation for these age groups accounting for potential 
confounders. Covariates included in the model were selected based on prior understanding of factors which might confound the relationship between an individual's age and the decision to transport a patient: ethnicity, gender, air bag deployment, whether the individual was trapped or ejected, and injury severity. Each of these covariates was represented using categorical variables. Injury severity was recorded on scene as one of five categories: death, disabling injury (injury preventing performance of normal activities), evident injury (obvious injury visible on scene), possible injury ("no visible injury, but person complains of pain") ${ }^{15}$. The purpose of these adjusted estimates was to obtain an estimate of the effect of age on the decision by patients and EMS providers to transport patients holding other factors constant.

In order to better understand the relationship between age and EMS transport rates, a second set of analyses were conducted using age in 39 categories of two year age groups (18-19, $20-21,22-23$, etc.) with $18-19$ as the reference group. Odds ratios were calculated to describe the relationship between individual variables and EMS transport. All analyses were conducted using SAS 9.2 (SAS Institute, Cary, North Carolina).

Three sensitivity analyses were performed. First, the relationship between patient age and EMS transportation was examined for each year of study data separately to examine temporal changes in the relationship. Second, analyses were repeated for the subset of people who were involved in collisions with a speed at impact of 35 miles (56.3 kilometers) per hour or more. Thirty-five miles (56.3 kilometers) per hour is the speed at which the United States National Highway Traffic Safety Administration performs full-frontal crash tests and is regarded by this group as a speed above which there is substantial potential for serious injury ${ }^{16}$. Third, the subset of patients with "possible injury" was examined to examine EMS decision making across the age span when injury severity is less evident.

\section{RESULTS}

In North Carolina in calendar years 2008-2011, there were 2,764,361 people involved in 1,113,958 MVC events, which could each include one or more cars (Figure 1). Of the 1,113,958 MVC events, $68.7 \%(\mathrm{n}=764,915)$ involved more than one car, $80.7 \%(\mathrm{n}=899,046)$ occurred on a dry road, and $68.0 \%(\mathrm{n}=757,411)$ occurred in daylight. Weather contributed to $4.9 \%(\mathrm{n}=54,984)$ of MVCs and drugs or alcohol were reported to be involved in $2.2 \%$ $(\mathrm{n}=24,247)($ Table 1$)$.

Of the 2,086,006 people aged 18-94 years involved in MVCs, $23.2 \%(\mathrm{n}=484,310)$ were seen by EMS and $8.4 \%(\mathrm{n}=175,768)$ were transported by EMS (Figure 1). MVCs were experienced by $1,892,692$ people aged $18-64$ years, 118,834 people aged $65-74$ years, 59,823 people aged $75-84$ years, and 14,657 people aged $85-94$ years. Older individuals were less likely to be drivers and more likely to be white, female, and either die or have a disabling or evident injury (Tables 2, 3).

Among those involved in MVC with EMS response, the unadjusted EMS transport rates for adults aged 18-64, 65-74, 75-84, and 85-94 years were 36.34\% (95\% Confidence Interval [CI] 36.20-36.48), 35.29\% (95\% CI 34.72-35.86), 36.26\% (95\% CI 35.48-37.04), and 
$37.95 \%$ (95\% CI 36.40-39.52), respectively. After adjustment for potential confounders, the EMS transport rates for adults aged 18-64, 65-74, 75-84, and 85-94 years were 36.02\% (95\% CI 35.88-36.16), 36.55\% (95\% CI 35.98-37.13), 37.31\% (95\% CI 36.53-38.10), and $38.22 \%$ (95\% CI 36.67-39.80), respectively. Visual examination of raw and adjusted EMS transportation rates by two year age group indicates that EMS transport rates were essentially unchanged with age until age 75 years, after which the rate increased by about $2 \%$ across the final two decades of lifespan analyzed (Figure 2).

Separate analysis of each of the four years from 2008-2011 showed the same trend in adjusted transport rates, a monotonic increase in transport rates with increasing age group. In MVCs with speed at impact of 35 miles (56.3 kilometers) per hour or more and EMS response, adjusted transportation rates showed similar transport rates across the age span: for age groups of 18-64, 65-74, 75-84, and 85-94 years transport rates were 42.30\% (95\% CI $42.10-42.50$ ), $42.11 \%$ (95\% CI 42.29-42.93), 43.10\% (95\% CI 41.98-44.24), and 44.33\% (95\% CI 42.00-46.67), respectively. In those with "possible injury" and EMS response, adjusted transportation rates showed a larger proportion transported but again only a very modest increase in transportation rates across the age span: for age groups of 18-64, 65-74, 75-84, and 85-94 years transport rates were $55.7 \%$ (95\% CI 55.4-55.8), 56.2\% (95\% CI 55.4-57.3), 57.6\% (95\% CI 56.2-58.9), and 59.1\% (95\% CI 56.4-61.8).

\section{DISCUSSION}

Across a large state with numerous EMS agencies, ambulance transport rates for adults seen by EMS after experiencing MVC were essentially stable with increasing patient age up to 75 years of age, after which there was a modest increase in the proportion transported. Most older adults experiencing MVC were not transported and the total increase in transportation rates between the oldest and youngest age groups was only about two percent. These results were robust to adjustments for collision and injury characteristics, analysis for individual study years, and among a subgroup of patients involved in high energy collisions. These results were contrary to our expectations.

Whether an individual is transported by EMS is a multifaceted decision that depends upon an assessment by EMS providers and patient willingness to be transported. Of patients evaluated but not transported by EMS, 20-28\% will later be seen in the $\mathrm{ED}^{17}$ and as many as $0.2 \%$ of trauma patients not transported by EMS subsequently die ${ }^{8}$. Compared to younger age groups, a higher proportion of older adults who present to the ED after being evaluated but not transported by EMS are admitted to the hospital. ${ }^{8}$ Our results suggest that even when comparing adults with experiencing similar collision and similar injury severity age is not an important factor in determining which patients are transported by EMS.

Our finding that only about $35 \%$ of older adults who experience MVC are transported following EMS evaluation indicates that a requirement to transport all adults aged 65 years or older would cause roughly a 3-fold increase in transports for this group of patients. Based upon data for North Carolina from 2008-2011, the latter scenario would result in more than 11,000 EMS transports of older MVC patients per year compared to the current number of about 4,000 per year. Even among the subgroup of patients experiencing MVCs with an 
impact speed of 35 miles (56.3 kilometers) per hour, the majority of patients are not transported. Thus, a requirement to transport all older adults seen by EMS after MVC would be costly and in most regions an unacceptable requirement for already over-burdened EMS systems. ${ }^{18}$

Field trauma triage criteria which identify patients of any age who need transport to a trauma center and which include vital signs, ${ }^{19,20}$ severity of vehicle damage, ${ }^{21}$ and use of antiplatelet or anticoagulation medications ${ }^{22}$ have been adopted by the Centers for Disease Control and Prevention (CDC $)^{23}$. A modified version of these criteria is currently used by EMS agencies in North Carolina. The North Carolina State EMS Multiple Trauma Protocol states that: "Geriatric patients should be evaluated with a high index of suspicion. Often occult injuries are more difficult to recognize and patients can decompensate unexpectedly with little warning." However, neither this protocol nor the CDC's field trauma triage criteria includes age as an explicit trigger for EMS transport. Further, these and other geriatric specific trauma triage criteria distinguish between patients who require trauma center evaluation vs. non-trauma center evaluation ${ }^{24,25}$. These criteria do not help EMS providers distinguish between older adults who require some form of additional medical evaluation vs. no further evaluation after trauma, and we were unable to find any published criteria which provide guidance in making this distinction. Whether it is possible to develop elderly-specific field triage criteria to identify patients needing medical evaluation that are more accurate than the current non-specific recommendation for a "high index of suspicion," which relies heavily on the judgment of EMS providers and patients, is unknown.

A second finding from this study with implications for the emergency care of older adults is that for both those patients transported and not transported by EMS, the frequency of evident or disabling injury was at least as high among older adults as among younger adults. Thus, the data do not support the assumptions that older adults experiencing MVC are often transported by EMS “just because they are old." These findings are consistent with the relatively high rates of acute pain observed in older patients presenting to the ED after $\mathrm{MVC}^{3}$. Among older adults experiencing MVC and not transported by EMS, 5\% had evidence of injury. Presumably some of these individuals obtain alternate means of transportation to the ED for evaluation shortly after the MVC. Outcomes for older patients who experience MVC but are neither transported by EMS nor independently seek medical care including the main morbid outcome from MVC, persistent pain and functional decline, are unknown.

In North Carolina, EMS agencies can collect compensation for completing transport to an ED but usually are not compensate for on-scene evaluation not resulting in transport. It is theoretically possible that this financial incentive might encourage transportation. However, most EMS services in North Carolina are municipality-based and not motivated by profit. Rather, both organizational leaders and individual providers focus on ensuring that EMS services are available for emergencies rather than maximizing transports.

Future research should seek to identify predictors of injury requiring EMS transport following MVC across all age groups. This will require both qualitative and quantitative studies. Qualitative studies should assess why patients are or are not being transported from 
the MVC scene following EMS response. Quantitative prospective studies in the form of cluster randomized trials or before-and-after studies should seek to link EMS data to outcomes to assess appropriateness of transport decisions and to identify predictors of injury at the MVC scene. All these results could be used to generate an age-specific protocol for evaluation and transport following MVC.

\section{LIMITATIONS}

There are several limitations to this study. MVCs that were not reported to the NC DMV by police on scene were not included in this analysis. It is unlikely that this affected our results because all MVCs with "death, injury or property damage exceeding $\$ 1,000$ must be reported to the Police" ${ }^{\text {26 }}$. Under-reporting of MVCs or failure to record EMS transports in the CRASH database might have biased our estimates, but such failures would be unlikely to vary by patient age and so are unlikely to influence the observed trends in EMS transport rates by age. Adjustment for important potential confounders allows us to examine the effect of age independent of other patient and collision characteristics and confirms that age had at most a small effect on transportation rates. However, NC DMV reports injury status as recorded by police on scene, which provides a blunt and imperfect characterization of injury severity; residual confounding by injury severity cannot be excluded. This could represent a differential misclassification of older adults with serious injuries; if this is the case, triage modification based on patient age is a potential solution. Patients who were dead on scene could not be reliably identified using the database. However, the number of patients dead on scene is small and unlikely to alter our estimates. Further, since older adults are more likely to be dead on scene than younger adults, ${ }^{27}$ including dead patients in the analysis and categorizing them as transported would tend to inflate transportation estimates for older patients. Accurately excluding all patients dead on scene would either have no effect on estimates or result in an even smaller difference in transport rates between younger and older adults. Although the data is verified for completeness and logical entries, we cannot assess the accuracy of data entry into the database. Additionally, we are not able to determine if the transport or non-transport was appropriate; rather, we provide an epidemiological perspective on transportation across the age-span.

We do not have information about the EMS care providers nor do we have the ability to examine the process by which EMS providers made decisions in the field. Characteristics of EMS providers, including gender, training level, and years of experience, could influence their decision to transport patients and were not included in this analysis ${ }^{28}$. Our study eliminated patients age 95 and older due to small sample size. As the population continues to age, this could be an important group of individuals experiencing MVC. Finally, our study is not a derivation of a decision instrument because we do not know which patients needed to be transported; the observed associations between patient characteristics and EMS transport rates are simply a description of existing care.

\section{CONCLUSION}

In North Carolina in calendar years 2008-2011, the difference in transport rates across the age span was small and the majority of older adults were not transported by EMS. Revisions 
to guidelines to reduce the occurrence of non-transportation of older adults with serious or life-threatening injuries after MVC will require a sophisticated decision instruments in order to avoid transportation of a large number of non- or minimally injured individuals.

\section{Acknowledgments}

Research reported in this publication was supported by the National Institute on Aging of the National Institutes of Health under Award Number K23AG038548 (Platts-Mills) and under Award Number 5-T35-AG038047-03 - UNC$\mathrm{CH}$ Summer Research in Aging for Medical Students (Hunold). This work is published with permission of the Traffic Records Branch of the the North Carolina Department of Transportation.

\section{References}

1. Bureau USC. Statistical Absstract of the United States. 2012. p. 693

2. [Accessed October 15th, 2013] Traffic Safety Facts, annual. 2012. at http://www.nrd.nhtas.dot/gov/ CATS/index.aspx

3. Platts-Mills TF, Hunold KM, Esserman DA, Sloane PD, McLean SA. Motor Vehicle Collisionrelated Emergency Department Visits by Older Adults in the United States. Acad Emerg Med. 2012; 19:821-7. [PubMed: 22724382]

4. Newgard CD. Defining the "older" crash victim: the relationship between age and serious injury in motor vehicle crashes. Accid Anal Prev. 2008; 40:1498-505. [PubMed: 18606283]

5. Lyman S, Ferguson SA, Braver ER, Williams AF. Older driver involvements in police reported crashes and fatal crashes: trends and projections. Injury prevention: journal of the International Society for Child and Adolescent Injury Prevention. 2002; 8:116-20. [PubMed: 12120829]

6. Clark DE, Winchell RJ, Betensky RA. Estimating the effect of emergency care on early survival after traffic crashes. Accident; analysis and prevention. 2013; 60C:141-7.

7. Phillips S, Rond PC 3rd, Kelly SM, Swartz PD. The failure of triage criteria to identify geriatric patients with trauma: results from the Florida Trauma Triage Study. J Trauma. 1996; 40:278-83. [PubMed: 8637079]

8. Staudenmayer K, Hsia R, Wang E, Sporer K, Ghilarducci D, Spain D, Mackersie R, Sherck J, Kline $\mathrm{R}$, Newgard C. The forgotten trauma patient: outcomes for injured patients evaluated by emergency medical services but not transported to the hospital. The journal of trauma and acute care surgery. 2012; 72:594-9. discussion 9-600. [PubMed: 22491541]

9. Schmidt T, Atcheson R, Federiuk C, Mann NC, Pinney T, Fuller D, Colbry K. Evaluation of protocols allowing emergency medical technicians to determine need for treatment and transport. Acad Emerg Med. 2000; 7:663-9. [PubMed: 10905645]

10. Schmidt TA, Atcheson R, Federiuk C, Mann NC, Pinney T, Fuller D, Colbry K. Hospital followup of patients categorized as not needing an ambulance using a set of emergency medical technician protocols. Prehosp Emerg Care. 2001; 5:366-70. [PubMed: 11642586]

11. Scheetz LJ. Effectiveness of prehospital trauma triage guidelines for the identification of major trauma in elderly motor vehicle crash victims. J Emerg Nurs. 2003; 29:109-15. [PubMed: 12660691]

12. Scheetz LJ. Trauma center versus non-trauma center admissions in adult trauma victims by age and gender. Prehospital emergency care: official journal of the National Association of EMS Physicians and the National Association of State EMS Directors. 2004; 8:268-72.

13. Knight S, Olson LM, Cook LJ, Mann NC, Corneli HM, Dean JM. Against all advice: an analysis of out-of-hospital refusals of care. Annals of Emergency Medicine. 2003; 42:689-96. [PubMed: 14581923]

14. Scheetz LJ. Prehospital factors associated with severe injury in older adults. Injury. 2010; 41:88693. [PubMed: 20542270]

15. DMV-349 Instruction Manual North Carolina Department of Transportation. 2012.

16. National Highway Traffic Safety Administration. The New Car Assessment Program Suggested Approaches for Furture Program Enhancements. 2007. 
17. Knight S, Olson LM, Cook LJ, Mann NC, Corneli HM, Dean JM. Against all advice: an analysis of out-of-hospital refusals of care. Ann Emerg Med. 2003; 42:689-96. [PubMed: 14581923]

18. Hatley T, Patterson PD. Management and financing of emergency medical services. N C Med J. 2007; 68:259-61. [PubMed: 17694845]

19. Lamantia MA, Stewart PW, Platts-Mills TF, Biese KJ, Forbach C, Zamora E, McCall BK, Shofer FS, Cairns CB, Busby-Whitehead J, Kizer JS. Predictive Value of Initial Triage Vital Signs for Critically Ill Older Adults. The western journal of emergency medicine. 2013; 14:453-60. [PubMed: 24106542]

20. Martin JT, Alkhoury F, O'Connor JA, Kyriakides TC, Bonadies JA. 'Normal' vital signs belie occult hypoperfusion in geriatric trauma patients. The American surgeon. 2010; 76:65-9. [PubMed: 20135942]

21. Jones IS, Champion HR. Trauma triage: vehicle damage as an estimate of injury severity. J Trauma. 1989; 29:646-53. [PubMed: 2724382]

22. Li J, Brown J, Levine M. Mild head injury, anticoagulants, and risk of intracranial injury. Lancet. 2001; 357:771-2. [PubMed: 11253975]

23. Sasser SM, Hunt RC, Faul M, Sugerman D, Pearson WS, Dulski T, Wald MM, Jurkovich GJ, Newgard CD, Lerner EB. Guidelines for field triage of injured patients: recommendations of the National Expert Panel on Field Triage, 2011. MMWR Recomm Rep. 2012; 61:1-20. [PubMed: 22237112]

24. Werman HA, Erskine T, Caterino J, Riebe JF, Valasek T. Members of the Trauma Committee of the State of Ohio EMSB. Development of statewide geriatric patients trauma triage criteria. Prehosp Disaster Med. 2011; 26:170-9. [PubMed: 22107767]

25. Ichwan B, Darbha S, Shah MN, Thompson L, Evans DC, Boulger CT, Caterino JM. GeriatricSpecific Triage Criteria Are More Sensitive Than Standard Adult Criteria in Identifying Need for Trauma Center Care in Injured Older Adults. Ann Emerg Med. 2014

26. In the Event of an Accident in North Carolina. NC Department of Motor Vehicles; 2011. at http:// www.dmv.com/nc/north-carolina/auto-accidents [Accessed October 12, 2013, 2013]

27. Kuhne CA, Ruchholtz S, Kaiser GM, Nast-Kolb D. Mortality in severely injured elderly trauma patients--when does age become a risk factor? World J Surg. 2005; 29:1476-82. [PubMed: 16228923]

28. Safdar B, Heins A, Homel P, Miner J, Neighbor M, DeSandre P, Todd KH. Impact of physician and patient gender on pain management in the emergency department--a multicenter study. Pain Med. 2009; 10:364-72. [PubMed: 18992042] 


\section{Highlights}

- We examined ambulance transport rates after motor vehicle collision.

- Of 484,310 adults seen by EMS, $36 \%$ were transported to emergency departments.

- Raw transport rates were only $2 \%$ higher for older vs. younger adults.

- Adjusted transport rates were also only $2 \%$ higher for older adults.

- Age-specific guidelines may improve the triage of older adults after MVC Abstract 


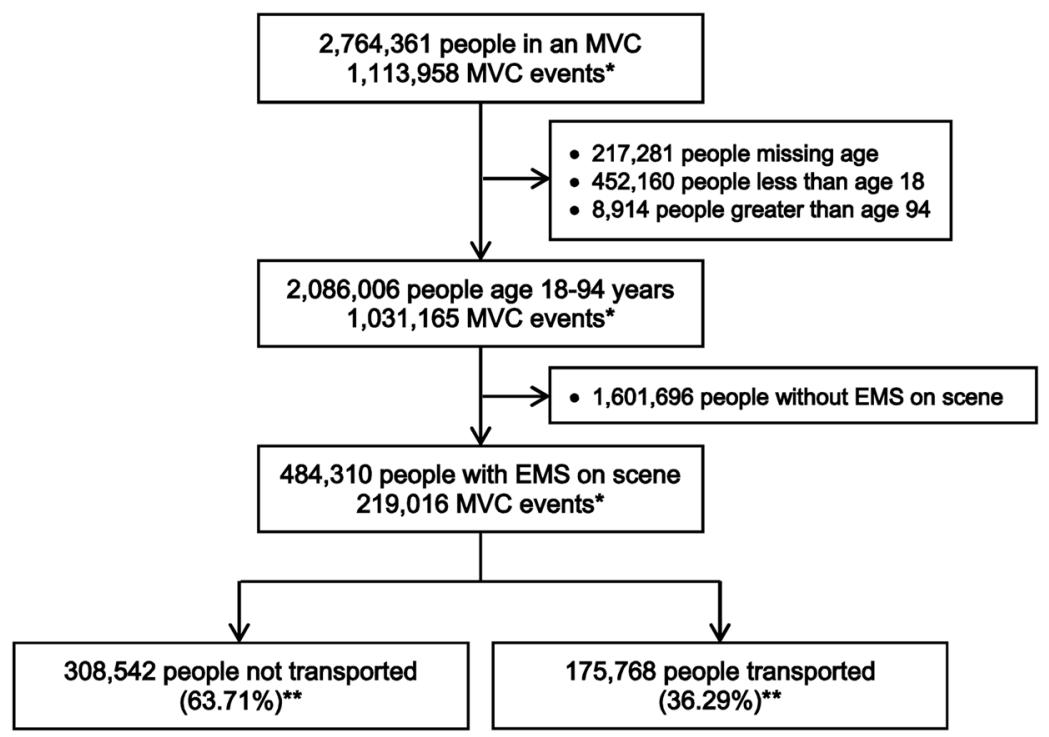

* An MVC event is one collision and may have involved one or more vehicles.

** Percentage of people of the 484,310 with EMS on scene.

Figure 1.

Flow diagram of the motor vehicle collision (MVC) population and sample analyzed in North Carolina for calendar years 2008-2011. 


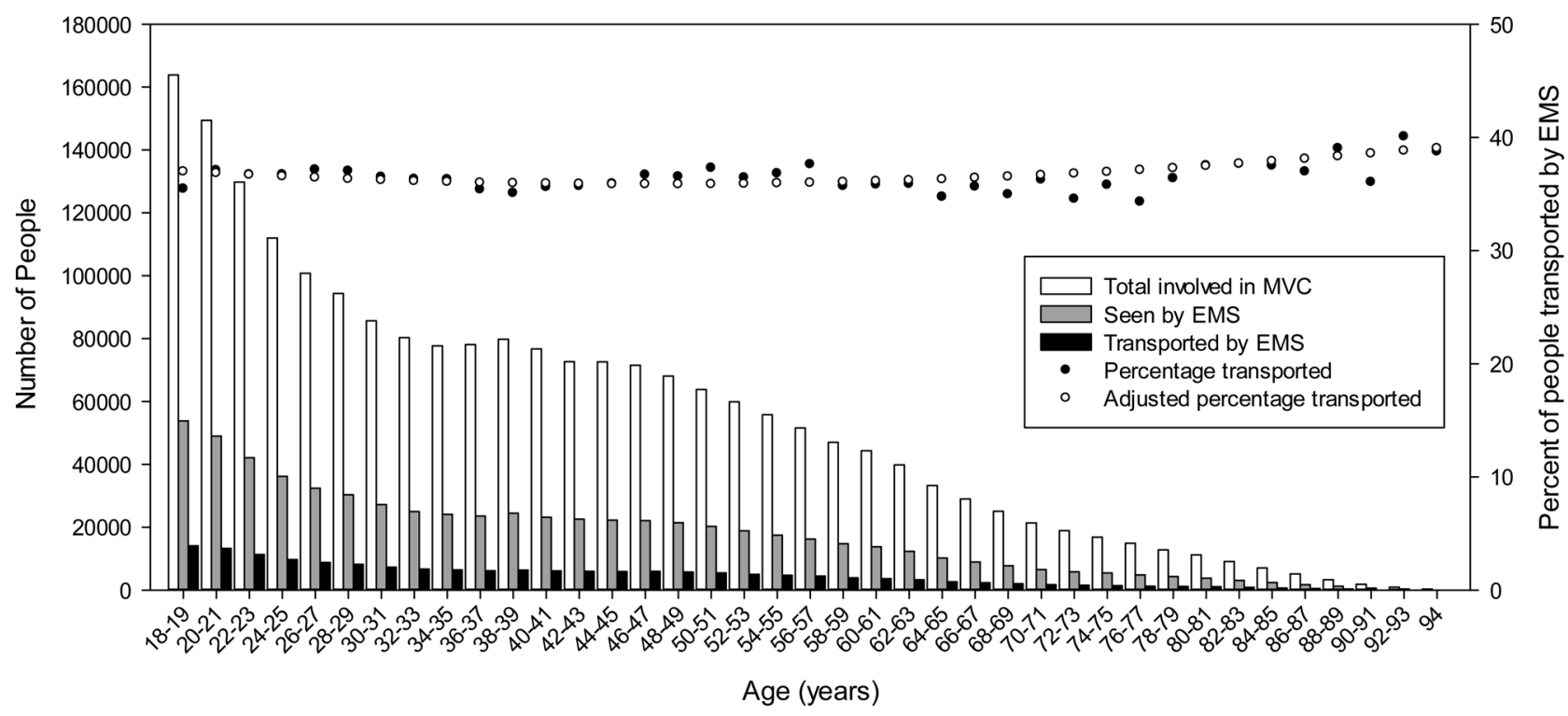

Figure 2.

Number of people aged 18-94 years $(n=2,086,006)$ involved in MVC, seen by EMS, and transported by EMS (vertical bars and left axis). Patients transported by EMS as a percentage of individuals seen by EMS after MVC with and without adjustment (circles and right axis). 


\section{Table 1}

Characteristics of motor vehicle collisions (MVCs) in North Carolina in calendar years 2008-2011. Information compiled by responders on scene through North Carolina Department of Motor Vehicles form DMV-349. All data reported as n (\%)

\begin{tabular}{|c|c|}
\hline Characteristic & $N=1,113,958$ \\
\hline \multicolumn{2}{|l|}{ Number of cars involved } \\
\hline 1 & $348,747(31.3)$ \\
\hline 2 & $708,297(63.6)$ \\
\hline 3 or more & $56,618(5.1)$ \\
\hline Missing & $296(<0.1)$ \\
\hline Involved $\geq 1$ non-motorist ${ }^{l}$ & $16,547(1.5)$ \\
\hline \multicolumn{2}{|l|}{ Crash locality } \\
\hline Rural ( $<30 \%$ developed) & $320,006(28.7)$ \\
\hline Mixed (30-70\% developed) & $169,130(15.2)$ \\
\hline Urban (>70\% developed) & $624,822(56.1)$ \\
\hline \multicolumn{2}{|l|}{ Road surface condition } \\
\hline Dry & $899,046(80.7)$ \\
\hline Wet & $171,747(15.4)$ \\
\hline Other ${ }^{2}$ & $40,873(3.7)$ \\
\hline Missing & $2,292(0.2)$ \\
\hline Weather contributed to MVC & $54,984(4.9)$ \\
\hline \multicolumn{2}{|l|}{ Ambient light } \\
\hline Daylight & $757,411(68.0)$ \\
\hline Dark & $350,237(31.4)$ \\
\hline Missing & $6,310(0.6)$ \\
\hline Alcohol/drug involvement ${ }^{3}$ & $24,247(2.2)$ \\
\hline \multicolumn{2}{|l|}{ Damage severity 4} \\
\hline Severe (5-7) & $85,210(7.6)$ \\
\hline Moderate (3-4) & $286,235(25.7)$ \\
\hline Mild (1-2) & $714,940(64.2)$ \\
\hline None $(0)$ & $22,162(2.0)$ \\
\hline Missing & $5,411(0.5)$ \\
\hline \multicolumn{2}{|l|}{ Damage cost estimate } \\
\hline$<\$ 1,000$ & $234,712(21.1)$ \\
\hline$\$ 1,001-\$ 5,000$ & $620,637(55,7)$ \\
\hline$>\$ 5,000$ & $245,539(22.0)$ \\
\hline Missing & $13,070(1.2)$ \\
\hline EMS response to MVC & $223,980(20.1)$ \\
\hline
\end{tabular}


${ }^{4}$ Recorded on scene on a severity score of $0-7$ 


\section{Table 2}

Characteristics of people involved in motor vehicle collisions in North Carolina in calendar years 2008-2011 by age group (years) $(n=2,086,606)$. Information compiled by law enforcement responders on scene. All data reported as $\mathrm{n}(\%)$ unless otherwise noted

\begin{tabular}{|c|c|c|c|c|}
\hline Characteristic & $\begin{array}{c}\text { Age 18-64 } \\
\mathrm{N}=1,892,692\end{array}$ & $\begin{array}{c}\text { Age 65-74 } \\
N=118,834\end{array}$ & $\begin{array}{l}\text { Age 75-84 } \\
\mathrm{N}=\mathbf{5 9 , 8 2 3}\end{array}$ & $\begin{array}{l}\text { Age 85-94 } \\
N=14,657\end{array}$ \\
\hline Age, mean (SD) & $36(13)$ & $69(3)$ & $79(3)$ & $88(2)$ \\
\hline \multicolumn{5}{|l|}{ Ethnicity } \\
\hline White & $1,161,869(61.4)$ & $90,827(76.4)$ & $49,104(82.1)$ & $12,433(84.8)$ \\
\hline Black & $530,261(28.0)$ & $23,622(19.9)$ & $9,218(15.4)$ & $1,940(13.2)$ \\
\hline Other & $191,786(10.1)$ & $3,870(3.3)$ & $1,202(2.0)$ & $206(1.4)$ \\
\hline Missing & $8,776(0.5)$ & $515(0.4)$ & $299(0.5)$ & $78(0.5)$ \\
\hline \multicolumn{5}{|l|}{ Gender } \\
\hline Male & $995,325(52.6)$ & $60,148(50.6)$ & 28,945 (48.4) & $7,038(48.0)$ \\
\hline Female & $894,417(47.3)$ & $58,517(49.2)$ & $30,765(51.4)$ & $7,593(51.8)$ \\
\hline Missing & $2,950(0.2)$ & $169(0.1)$ & $113(0.2)$ & $26(0.2)$ \\
\hline \multicolumn{5}{|l|}{ Air Bag Deployment } \\
\hline Deployed & $172,018(9.1)$ & $9,291(7.8)$ & $5,314(8.9)$ & $1,416(9.7)$ \\
\hline Not deployed & $1,448,809$ (76.6) & $94,326(79.4)$ & $47,342(79.1)$ & $11,151(76.1)$ \\
\hline No air bags & $252,597(13.4)$ & $14,214(12.0)$ & $6,589(11.0)$ & $1,933(13.2)$ \\
\hline Missing & $19,268(1.0)$ & $1,003(0.8)$ & $578(1.0)$ & $157(1.1)$ \\
\hline \multicolumn{5}{|l|}{ Trapped } \\
\hline Yes & $17,194(0.9)$ & $1,425(1.2)$ & $824(1.4)$ & $241(1.6)$ \\
\hline No & $1,838,938(97.2)$ & $115,475(97.2)$ & $58,050(97.0)$ & $14,193(96.8)$ \\
\hline Missing & $36,560(1.9)$ & $1,934(1.6)$ & 949 (1.6) & $223(1.5)$ \\
\hline \multicolumn{5}{|l|}{ Ejected } \\
\hline Totally & $18,373(1.0)$ & $660(0.6)$ & $153(0.3)$ & $46(0.3)$ \\
\hline Partially & $2,553(0.1)$ & $114(0.1)$ & $34(0.1)$ & $2(<0.1)$ \\
\hline No & $1,834,298(96.9)$ & $116,145(97.7)$ & $58,693(98.1)$ & $14,391(98.2)$ \\
\hline Missing & $37,468(2.0)$ & $1,915(1.6)$ & $943(1.6)$ & $218(1.5)$ \\
\hline EMS on scene & $438,978(23.2)$ & $27,021(22.7)$ & $14,601(24.4)$ & $3,710(25.3)$ \\
\hline Transported by EMS & $159,529(8.4)$ & $9,537(8.0)$ & $5,294(8.9)$ & $1,408(9.6)$ \\
\hline \multicolumn{5}{|l|}{ Injury status } \\
\hline Death & $4,184(0.2)$ & $375(0.3)$ & $335(0.6)$ & $113(0.8)$ \\
\hline Disabling & $8,190(0.4)$ & $491(0.4)$ & $252(0.4)$ & $50(0.3)$ \\
\hline Evident & $78,868(4.2)$ & $4,466(3.8)$ & $2,641(4.4)$ & $770(5.3)$ \\
\hline Possible & $266,860(14.1)$ & $15,766(13.3)$ & $7,646(12.8)$ & $1,889(12.9)$ \\
\hline None & $1,509,466(79.8)$ & $96,356(81.1)$ & $48,175(80.5)$ & $11,656(79.5)$ \\
\hline Missing & $25,124(1.3)$ & $1,380(1.2)$ & 774 (1.3) & $179(1.2)$ \\
\hline
\end{tabular}




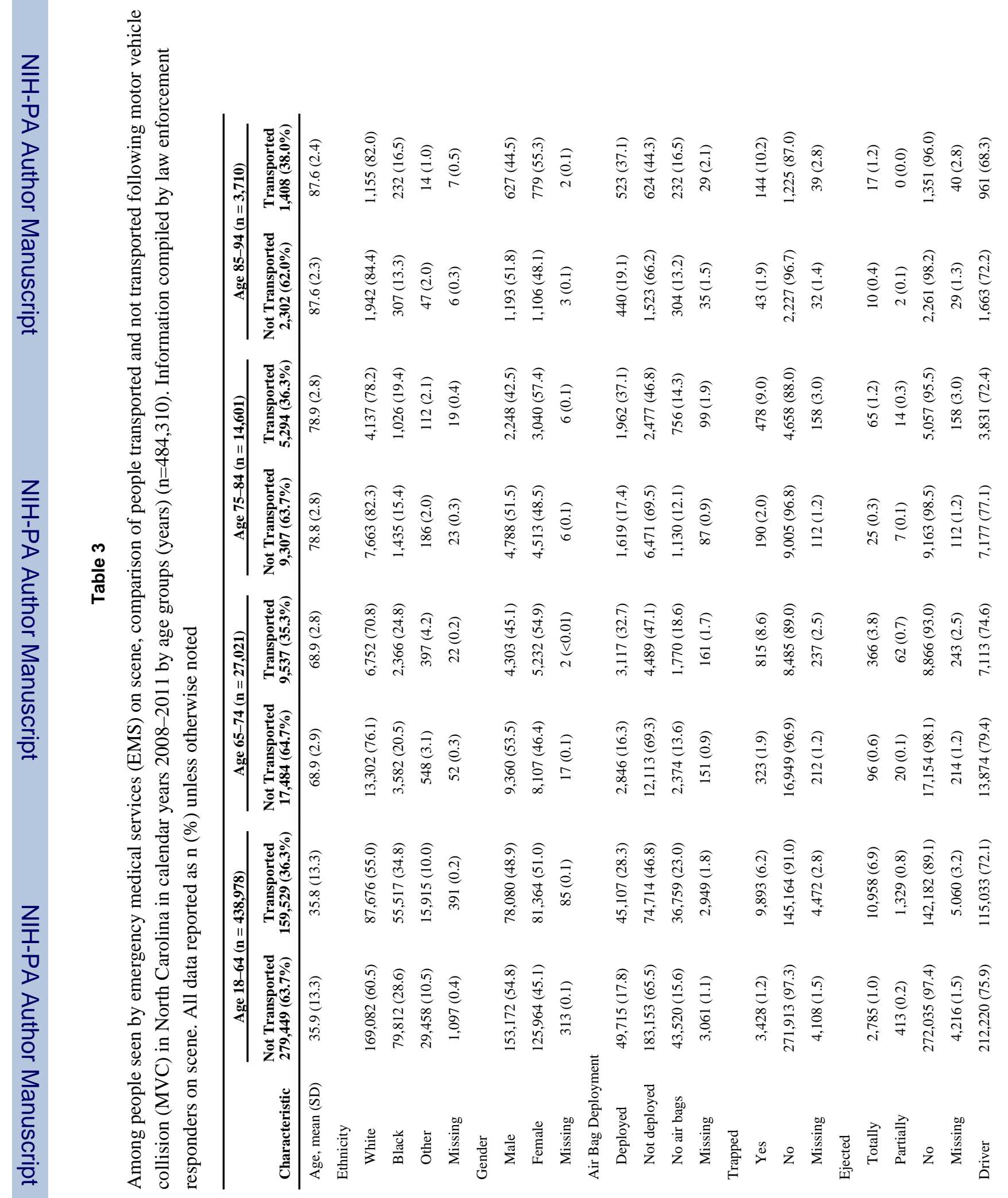


